\title{
10-user, truly-asynchronous OCDMA experiment with 511- chip SSFBG en/decoder and SC-based optical thresholder
}

\author{
Xu Wang and Naoya Wada \\ Information and Network Systems Department, National Institute of Information and Communication Technology (NICT), \\ 4-2-1 Nukui-Kitamachi, Koganei, Tokyo 184-8795 Japan. xwang@nict.go.jp \\ Taro Hamanaka and Ken-ichi Kitayama \\ Department of Electronics and Information Systems, Osaka University, 2-1 Yamadaoka, Suita, Osaka 565-0871, Japan
}

\author{
Akihiko Nishiki \\ Advanced Devices Laboratories, Corporate Research \& Development Center, Oki Electric Industry Co., 193-8550 Tokyo, Japan
}

\begin{abstract}
We demonstrate for the first time a 10-user, truly-asynchronous, gigabit OCDMA experiment over $50 \mathrm{~km}$ transmission using 511-chip SSFBG encoder/decoder and supercontinuum -based optical thresholder. Suppression of both beat noise and MAI are keys to the success.

(C) 2004 Optical Society of America
\end{abstract}

OCIS codes: (060.2330) Fiber optics communication (060.4230) Multiplexing (060.4370) Nonlinear optics, fibers

Introduction The passive optical network (PON) is promising for FTTH systems. Optical code division multiple access (OCDMA) is one promising candidate for next-generation broadband multiple access technique attributing to full asynchronous transmission, low latency access as well as soft capacity on demand [1-7]. There are several different OCDMA implementations that could be roughly classified according to operation principle as incoherent and coherent OCDMA [1]. Recently, coherent OCDMA using ultra-short optical pulse is receiving increasing attention with the progress of reliable and compact encoder/decoder devices, such as spatial light phase modulator (SLPM), planar lightwave circuit (PLC) and superstructured fiber Bragg grating (SSFBG). In coherent OCDMA, encoding and decoding are based on optical field amplitude instead of power intensity. The coding can be either direct time-spreading the ultra-short optical pulse using PLC [2] and SSFBG [7-8] or spectral phase-encoded timespreading using SLPM [3-6].

In a common multi-user OCDMA network, multiple access interference (MAI) noise is the main issue [1-7]. The MAI could be suppressed effectively by employing time gating [2] or optical thresholding techniques [3-6]. Recently, multi-user coherent OCDMA experiments have been demonstrated by utilizing optical thresholding based on second harmonic generation (SHG) in periodically-poled lithium niobate (PPLN) [3, 4] and nonlinear effect in high nonlinear fiber (HNLF) [5, 6] to significantly suppress the MAI noise.

However, coherent OCDMA could suffer from severe signal-interference (SI) beat noise if the signal and interferences overlap each other. The SI beat noise, which dominates over the MAI noise in such system, eventually limits the maximum number of active users that can be supported in the network [1]. Unfortunately, the SI beat noise could not be suppressed effectively by optical thresholding as it accompanies with the recovered signal pulse. Therefore, either slot-level [3, 6-7] or chip-level [4-6] timing coordination has been applied in previous experiments to enable multi-user transmission. Slot-level coordination is a rough synchronous approach that the signal and interferences are intentionally separated in time, therefore no beat noise will arise. Chip-level coordination is a precise synchronous approach that the signal and each interference have to be precisely aligned on a chip-level with zero interference to mitigate the beat noise. Both of them are synchronous approaches that sacrifice the most desired characteristic of OCDMA: "asynchronism”. Besides, the former one significantly lowers the frequency efficiency of the system, while the latter one requires very strict network synchronization ( ps) that is almost impossible in practical. For practical OCDMA network application, the capability of asynchronous multi-user access is essential.

In this paper, we will report, for the first time, a truly-asynchronous 10-user gigabit coherent OCDMA transmission experiment in the presence of beat noise as well as MAI without any timing coordination.

System design In an asynchronous OCDMA network with K active users, the received signal of target user could overlap with the K-1 interferences from undesired users asynchronously as illustrated in the bottom-right inset of Fig.1. Therefore, suppression of the SI beat noise as well as the MAI are crucial. One of the effective approaches is employing ultra-long optical code (OC) with uniform cross-correlations to lower the interference level $\xi$. A theoretical analysis has predicted that to support up to $\mathrm{K}=10$ error free $\left(\mathrm{BER}<10^{-9}\right)$ transmission with chip-rate detection, $\xi$ should be lower than $-27 \mathrm{~dB}$ [1]. Phase-shifted SSFBG en/decoder is one desired candidate that has the 


\section{PDP33}

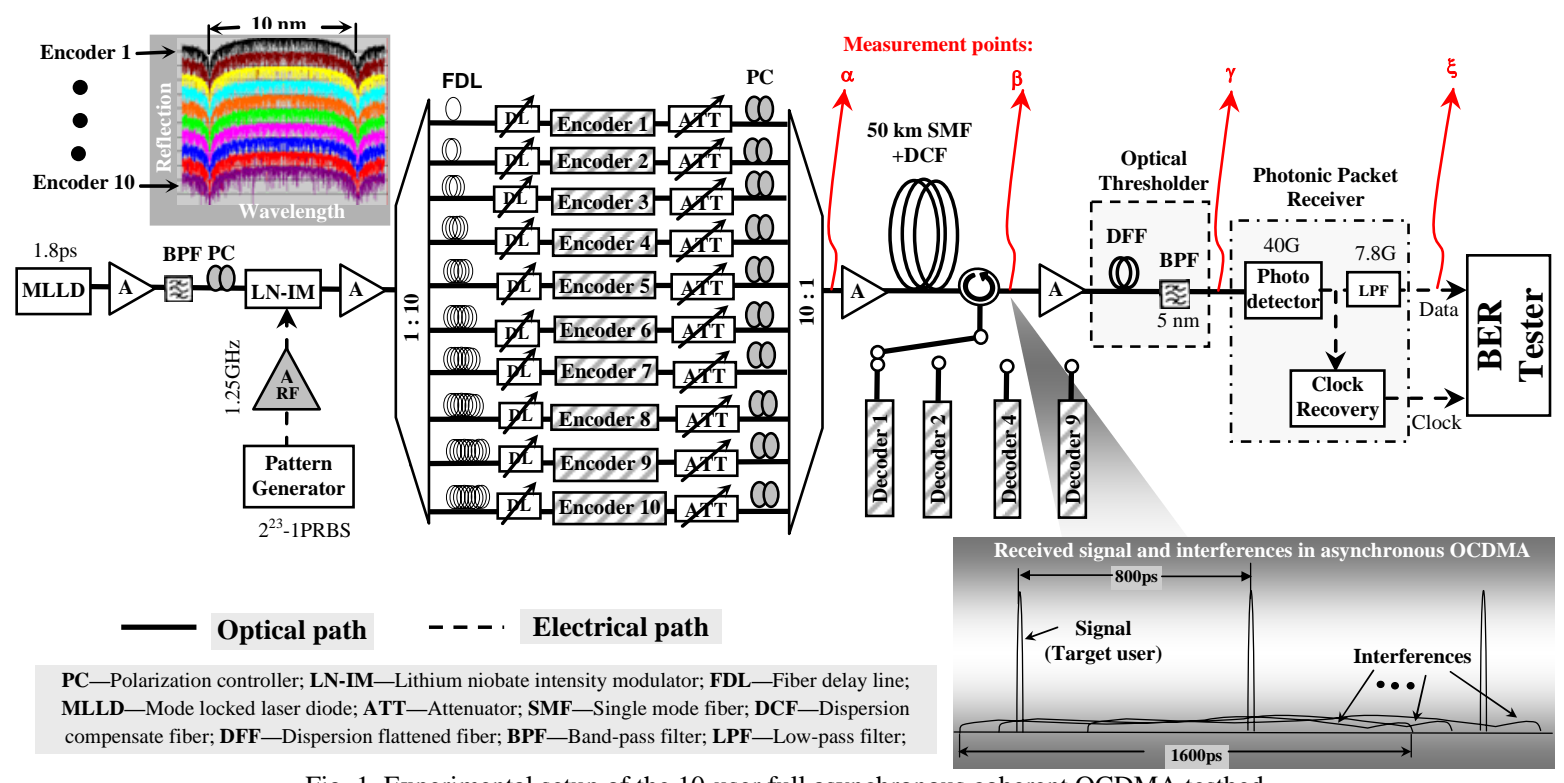

Fig. 1. Experimental setup of the 10-user full asynchronous coherent OCDMA testbed

capability to process OC as long as 511-chip with chip-rate as high as $640 \mathrm{Gchip} / \mathrm{s}$ [8] enabling us to challenge a truly-asynchronous OCDMA. In theory, about 7 active users can be supported for error free transmission at 1.25 Gbit/s with data-rate detection, while by employing optical thresholding to reject the MAI, the number of users could be doubled [1].

Experiments Figure 1 shows the experimental setup of our 10-user asynchronous coherent OCDMA testbed. An optical pulse train with 1.8 ps pulsewidth was generated by the mode-locked laser diode (MLLD) and modulated by $2^{23}-1$ pseudo-random bit sequence (PRBS) at $1.25 \mathrm{Gbit} / \mathrm{s}$. The amplified signal was equally split into ten arms and encoded by 10 different encoders. The encoders are 511-chip, 640 Gchip/s SSFBGs whose frequency responses are shown in the top-left inset of Fig. 1. Codes 1 10 are carefully chosen from 511-chip binary-phase-shift-key Gold codes so as to have relatively low aperiodic cross-correlations [8]. Fixed fiber delay lines with different lengths are inserted in the ten arms to randomly set the time delays and de-coherence signals of different users. Tunable optical delay lines (DL) are inserted as well to investigate the impact of different phases of signal-interference overlapping. In a practical PON environment, the polarization states of the signals may be random. However, for investigating the system performance in the worst scenario that the interference becomes most serious, polarization controllers (PC) are placed to align the polarization states of all signals. Besides, tunable attenuators (with switches) are used in all arms to balance the power levels from the 10 users and adjust the number of active users.

The 10-user OCDMA signals are mixed, amplified and launched into $50 \mathrm{~km}$ single mode fiber. Dispersion compensation fiber (DCF) is employed to compensate the transmission dispersion. The encoded asynchronous multi-user OCDMA signals at point $\alpha$ are shown in the top of Fig. 2(a) for different number of users (K). The duration of encoded waveform ( $\sim 800 \mathrm{ps})$ is slightly shorter than that of one bit ( 804 ps). Therefore, as illustrated in the bottom-right inset of Fig. 1, signal overlaps with interferences completely in this asynchronous experiment, no blank time-slot has been reserved for timing coordination as it has been done in previous approaches [3-7].

At the receiver side, four SSFBG decoders were employed to recover signals from users 1,2 , 4 and 9 . The eye diagrams of the decoded signals from decoder 4 measured at point $\beta$ are shown in the middle of Fig. 2(a). It is obvious that the increase of MAI and SI beat noise with the increase of K. Figure 2(b) shows the SHG traces of the decoded signals for single- and 10-user. The pulse widths are measured to be 2.2 and 3.0 ps, respectively. The high level pedestal and the broader pulsewidth of the pulse in 10-user case are mainly due to the MAI noise and the SI beat noise, respectively. In the experiment, we employed a novel optical thresholding by utilizing super-continuum (SC) generation in a normal dispersion-flattened-fiber (DFF) [9]. This SC-based optical thresholding is characterized by the polarization-independency and rather low insertion loss (13 $\mathrm{dB})$, compared to other techniques. The average operation power is about 1.4 and $10.3 \mathrm{dBm}$ for $\mathrm{K}=1$ and 10 , respectively. This means the peak power for single user is about $6.3 \mathrm{~W}$. This is higher than PPLN ( 1W), but is the lowest one among fiber-based optical thresholdings [9]. The eye diagrams and SHG traces measured at point $\gamma$ are shown as well in Figs. 2(a) (bottom) and (b), respectively. It is clear seen that the MAI noise has been eliminated effectively, while the SI beat noise still 


\section{PDP33}

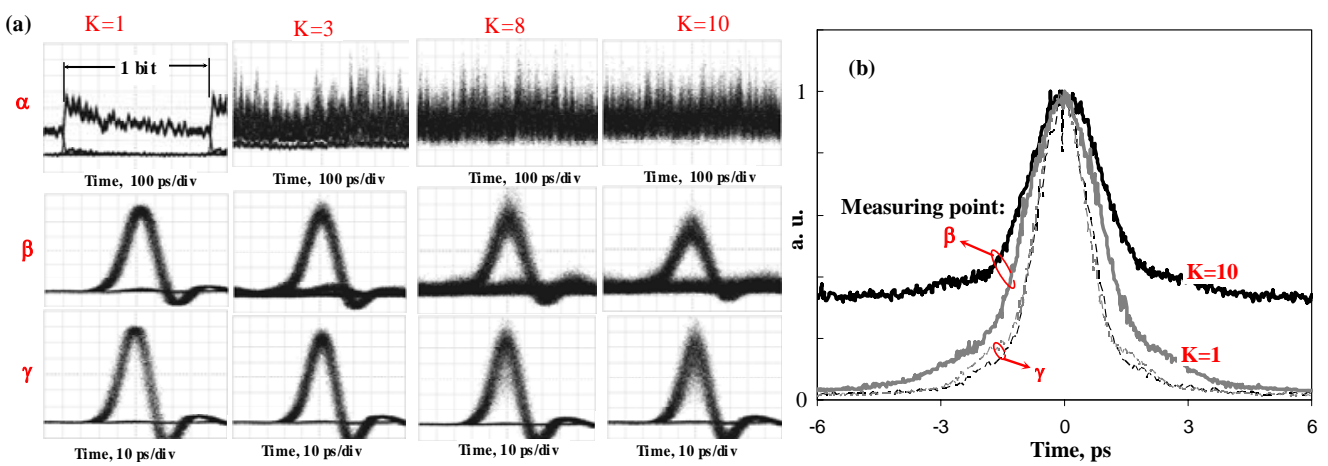

Fig. 2. (a) Measured eye diagrams at points $\alpha, \beta$ and $\gamma$; (b) SHG traces of decoded signals before and after SC-based OT

remains. The pulse widths are measured to be $\sim 1.5$ ps for both $\mathrm{K}=1$ and 10 showing that pulse reshaping has been done as well with this SC-based optical thresholding. Finally, an optical packet receiver followed by a $7.8 \mathrm{GHz}$ lowpass-filter (LPF) was employed in the testbed to perform data-rate detection for the signal.

BERs shown in Fig. 3(a) were measured with different $\mathrm{K}$ for both back-to-back (B-to-B) and $50 \mathrm{~km}$ transmission on this testbed. In the 10-user experiment, the OCDMA signals were mixed in a truly-asynchronous manner with random time delays, random data patterns, random bit phases, and random polarization states. We have also tested in the worst scenario: bit synchronous and aligned polarization state. In all these cases, error-free transmission has been achieved for all 4 receivers. This fact confirms that the SI beat noise and the MAI have been suppressed effectively as predicted by the theory. The eye diagrams for the four receivers measured at point $\xi$ in the worst scenario are shown in Fig. 3(b). Figure 3(c) shows average power penalty against $\mathrm{K}$ for $\mathrm{BER}=10^{-9}$ together with theoretical calculations. The deviations between experiment and theory are mainly due to the non-ideal optical thresholding.

In addition, we also measured the BERs w/o the SCbased optical thresholding for all the cases. The average power penalties are plotted in Fig. 3(c) as well with the theoretical calculations. In the B-to-B case, up to 7 users error free has been achieved agreeing with the theory prediction, while after $50 \mathrm{~km}$ transmission, only 6 users has been achieved, which is presumably due to the signal distortion during transmission. The experimental results agree with theoretical result very well.

Conclusions 10-user, truly-asynchronous, gigabit coherent OCDMA transmission experiment has been successfully demonstrated over $50 \mathrm{~km}$ SMF without any timing coordination. The key enabling techniques are 511-chip SSFBG encoder/decoder as well as SC-based optical thresholding. Further improvement of the optical thresholding will enable us to accommodate more users.

\section{References}

1. X. Wang and K. Kitayama, J. Lightwave Technol, 22, 2226-2235 (2004).

2. H. Sotobayashi, et. al., IEEE Photon. Tech.Lett., 14, 555-557 (2002).

3. Z. Jiang, et. al., IEICE Electron. Express, 1,398-403 (2004).

4. Z. Jiang, et. al., J. Lightwave Technol, 23, 143-158 (2005).

5. R. P. Scott, et. al., IEEE Photon. Tech. Lett., 16, 2186-2188, (2004).

6. V. J. Hernandez, et. al., J. Lightwave Technol, 22, 2671-2679 (2004).

7. P. C. Teh, et. al., OFC’02, ThEE1, 2002.

8. X. Wang, et. al., Optics Lett., 30, 355-357 (2005).

9. X. Wang et. al., submitted to OECC’05.
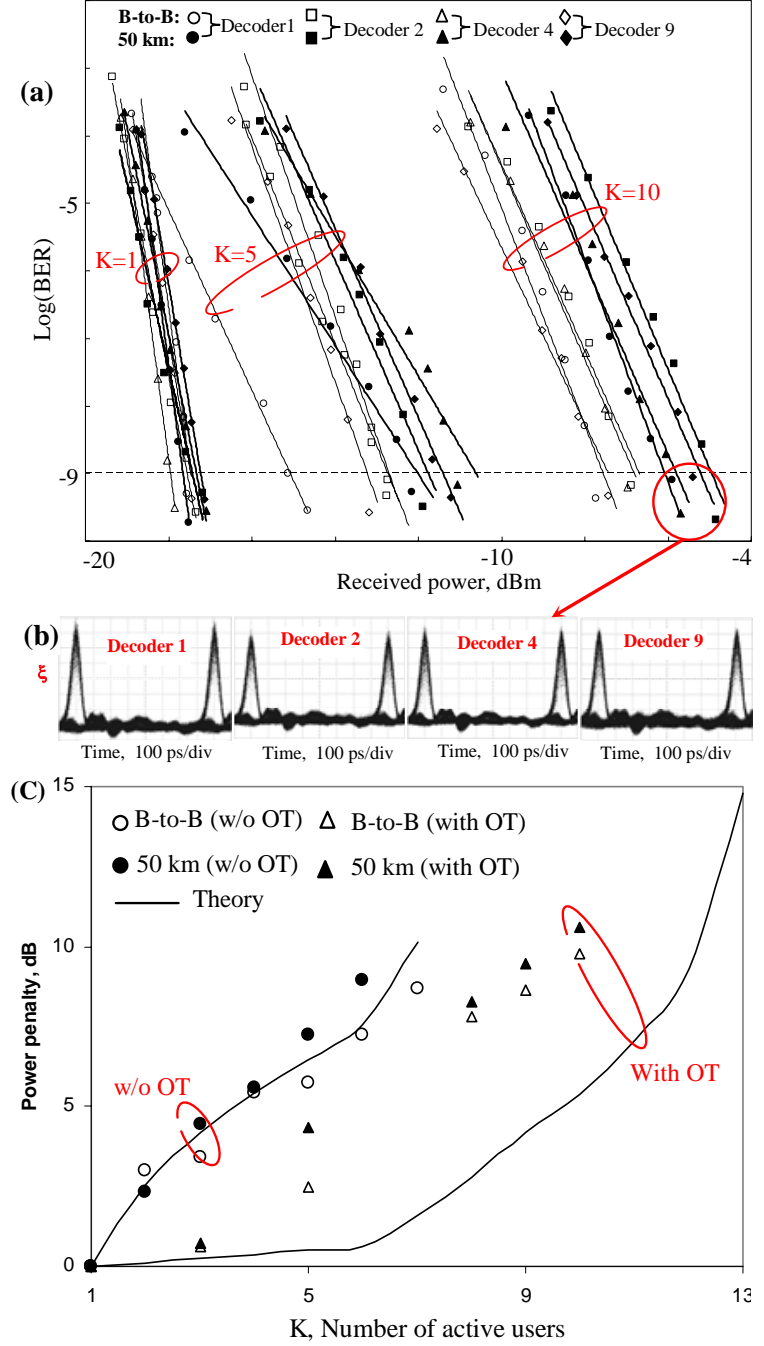

Fig. 3. (a) Measured BERs; (b) Eye diagrams from four decoders with $\mathrm{K}=10$ and $50 \mathrm{~km}$ transmission; (c) Power penalty vs. $\mathrm{K}$ 